\title{
Hydrogenotrophic methanogens are the key for a successful bioaugmentation to alleviate ammonia inhibition in thermophilic anaerobic digesters
}

Tian, Hailin; Yan, Miao; Treu, Laura; Angelidaki, Irini; Fotidis, Ioannis

Published in:

Bioresource Technology

Link to article, DOI:

10.1016/j.biortech.2019.122070

Publication date:

2019

Document Version

Peer reviewed version

Link back to DTU Orbit

Citation (APA):

Tian, H., Yan, M., Treu, L., Angelidaki, I., \& Fotidis, I. (2019). Hydrogenotrophic methanogens are the key for a successful bioaugmentation to alleviate ammonia inhibition in thermophilic anaerobic digesters. Bioresource Technology, 293, [122070]. https://doi.org/10.1016/j.biortech.2019.122070

\section{General rights}

Copyright and moral rights for the publications made accessible in the public portal are retained by the authors and/or other copyright owners and it is a condition of accessing publications that users recognise and abide by the legal requirements associated with these rights.

- Users may download and print one copy of any publication from the public portal for the purpose of private study or research.

- You may not further distribute the material or use it for any profit-making activity or commercial gain

- You may freely distribute the URL identifying the publication in the public portal 


\section{Hydrogenotrophic methanogens are the key for a successful}

\section{2 bioaugmentation to alleviate ammonia inhibition in thermophilic}

\section{3 anaerobic digesters}

4

5 Hailin Tian ${ }^{\text {a, b }}$, Miao Yan ${ }^{\text {a }}$, Laura Treu ${ }^{\text {a c c }}$, Irini Angelidaki ${ }^{\text {a }}$, Ioannis A. Fotidis ${ }^{\text {a, * }}$

6

7 a Department of Environmental Engineering, Technical University of Denmark,

8 Bygningstorvet Bygning 115, 2800 Kgs. Lyngby, Denmark

9

$10{ }^{\mathrm{b}}$ Environmental Research Institute, National University of Singapore, 1 Create Way,

11 Create Tower, Singapore 138602, Singapore

12

$13{ }^{\mathrm{c}}$ Department of Biology, University of Padua, 35131 Padua, Italy

14

$15 *$ Corresponding Author: Ioannis A. Fotidis, Department of Environmental Engineering,

16 Technical University of Denmark, Bygningstorvet Bygning 115, DK-2800 Kgs.

17 Lyngby, Denmark, Phone: (+45) 45251418; Fax: (+45) 45933850; e-mail:

18 ioanf@env.dtu.dk 


\section{Abstract}

Bioaugmentation to alleviate ammonia inhibition under thermophilic anaerobic

21 digestion has never been reported, as well as the working mechanism that allows a fast

22 and successful bioaugmentation. Thus two bioaugmentation inocula (an enriched

23 culture, and a mixed culture composed 50/50 by Methanoculleus thermophilus and the

24 enriched culture) on the recovery of ammonia-inhibited thermophilic continuous

25 reactors was assessed. The results showed that bioaugmentation improved methane

26 yield by $11-13 \%$ and decreased the volatile fatty acids (VFA) by $45-52 \%$ compared to

27 the control reactor (abiotic augmentation). Moreover, the importance of

28 hydrogenotrophic methanogens to a fast and successful bioaugmentation was

29 recognized. Specifically, the instant hydrogen partial pressure reduction by the

30 bioaugmented hydrogenotroph created thermodynamically favourable conditions for the

31 acetate oxidation process and consequently, the catabolism of other VFA. High-

32 throughput sequencing results strengthened this explanation by showing that the

33 bioaugmented $M$. thermophilus stimulated the growth of syntrophic acetate oxidising

34 bacterium Thermacetogenium phaeum, immediately after bioaugmentation.

\section{Keywords}

36 Biogas; Ammonia-tolerant consortium; Microbial community; Methanoculleus

37 thermophilus; Methanosarcina thermophila. 


\section{Introduction}

Many countries have recently introduced policies, aiming to increase the share of renewable energy in the total national energy consumption. Methane, a renewable energy carrier, produced through anaerobic digestion (AD) of organic materials, plays an important role in renewable energy supply (Kamali et al., 2016). Many nitrogen-rich biomasses such as chicken manure, aquaculture waste, slaughterhouse waste, microalgae, etc., are attractive AD feedstocks because of their high methane potentials (Tian et al., 2018b). The digestate from the AD of nitrogen-rich substrates is also classified as valuable biofertilizer due to its high levels of plant-available $\mathrm{N}$ nutrient (Möller and Müller, 2012). However, poor reactor performance with low methane production efficiency has been reported when nitrogen-rich substrates were used (Rajagopal et al., 2013). Within Europe Union (EU), there are more than 16,000 biogas plants with total installed electricity capacity higher than 10,100 MW (Scarlat et al., 2018). It was reported that some of these biogas plants lost more than $30 \%$ of their methane potential and consequently of their energy production, due to the high ammonia levels $\left(>3.0 \mathrm{~g} \mathrm{NH}_{4}{ }^{+}-\mathrm{N} \mathrm{L}^{-1}\right)$ formed from the degradation of nitrogen-rich substrates (Angelidaki et al., 2005). In other words, the biogas industry is suffering significant economic losses due to ammonia problem. Therefore, it is urgent to develop a practical method to overcome ammonia inhibition and improve the energy production efficiency of the biogas reactors.

$\mathrm{AD}$ is mediated by different microorganisms, and is typically described as a four steps process, i.e. hydrolysis, acidogenesis, acetogenesis and methanogenesis

60 (Angelidaki et al., 2011). The complex polymers are hydrolysed into different monomers during hydrolysis and consequently converted into different volatile fatty 
acids (VFAs) during acidogenesis. Then acetate is mainly formed during acetogenesis from other VFAs, such as propionate (Eq. (1)) and butyrate (Eq. (2)). The electrons produced during the oxidation of propionate and butyrate are transferred to protons to generate hydrogen. It must be mentioned that these oxidation reactions are thermodynamically unfavourable and can only happen when the produced hydrogen is consumed by methanogens to keep a low level. Finally, methane is produced in methanogenesis from either oxidation of acetate (Eq. (5)) or reduction of $\mathrm{CO}_{2}$ by $\mathrm{H}_{2}$ (Eq. (4)), which is also identified as the most sensitive step to ammonia compared to other steps (Tian et al., 2018a). Acetate can be metabolized to methane in two ways: syntrophic acetate oxidation coupled with hydrogenotrophic methanogenesis (SAOHM) (Eqs. (3) and (4)) and aceticlastic methanogenesis (AM) (Eq. (5)). Moreover, SAO-HM is believed to be more robust compared to AM at high ammonia levels (Sun et al., 2014; Werner et al., 2014; Tian et al., 2018a). and free ammonia $\left(\mathrm{NH}_{3}, \mathrm{FAN}\right)$ in the aqueous solutions. FAN, which increases along with $\mathrm{pH}$ and temperature, is the most toxic form of ammonia for the AD process (Massé 
et al., 2014). Thus, at the same TAN levels, thermophilic $\left(45-65^{\circ} \mathrm{C}\right) \mathrm{AD}$ process usually faces much higher ammonia stress compared to mesophilic $\left(30-40^{\circ} \mathrm{C}\right)$ process (Hansen et al., 1998). However, even with this drawback, thermophilic AD process still gains more and more attention in recent years, because of many advantages over mesophilic AD process. For example, thermophilic microorganisms usually have higher metabolic rates and higher growth rates compared to mesophilic ones, consequently result in shorter hydrolytic retention time (HRT) and higher organic matter removal rate (Weiland, 2010). Most importantly, thermophilic reactors are able to destruct the pathogenic microorganisms without the need of a pasteurization unit, which is necessary for the mesophilic reactors (Kim et al., 2002).

In recent years, bioaugmentation has been reported to alleviate ammonia inhibition in mesophilic continuously stirred tank reactor (CSTR) (Fotidis et al., 2014; Fotidis et al., 2017; Tian et al., 2019a). However, the basic bioaugmentation working mechanism and the microbiological interactions that allow a fast and successful bioaugmentation have not been clearly identified yet. Fotidis et al. (2014) proposed a "microbiological

100 domino effect" theory, which the bioaugmentation culture triggered the growth of other 101 ammonia-tolerant microorganisms. However, a solid microbial community dynamics during the ammonia alleviation process still needs to be investigated due to the lack of detailed bacterial information in the aforementioned study. The effect of the

104 bioaugmentation culture on the indigenous microbial community of the reactors is still 105 not clear so far, especially for the different bioaugmentation performance cause by the 106 different bioaugmentation inocula. Additionally, bioaugmentation in thermophilic 107 reactors is still a challenge compared to mesophilic reactors due to the higher ammonia 108 stress, the higher sensitivity to the changes of the environment, and the lack of available 
thermophilic ammonia-tolerant consortia, etc. (Yenigün and Demirel, 2013). Recently, a

110 successful acclimatization to high ammonia levels (TAN=5.0 $\mathrm{g} \mathrm{NH}_{4}{ }^{+} \mathrm{L}^{-1}, \mathrm{FAN}=1.4 \mathrm{~g}$

$111 \mathrm{NH}_{3} \mathrm{~L}^{-1}$ ) of a thermophilic methanogenic consortium (mainly Methanosarcina

112 thermophile, 95\% relative abundance among the methanogens) and a pure

113 hydrogenotrophic methanogenic strain (Methanoculleus thermophilus), was reported

114 (Tian et al., 2018a; Tian et al., 2019b). This creates the opportunity to compare the

115 different bioaugmentation inocula and decipher the interactions and/or the mechanisms

116 that drive and define a successful bioaugmentation process.

117 Based on the above, the main goal of the current study was to identify the basic

118 bioaugmentation working mechanism and analyse the microbial keystones of a fast and

119 successful bioaugmentation. In order to realise the main goal, two preliminary goals

120 were also considered: 1) to achieve, for the first time, successful bioaugmentation in

121 thermophilic CSTR reactors and 2) to compare the performance and interactions of

122 different bioaugmentation inocula. To realize these goals, two different ammonia-

123 tolerant cultures (i.e. an enriched culture, and a mixed culture composed by $M$.

124 thermophilus and the enriched culture) were used as bioaugmentation inocula to

125 alleviate ammonia toxicity in different thermophilic CSTR reactors.

\section{$126 \quad 2 \quad$ Material and methods}

\section{$127 \quad 2.1 \quad$ Inoculum and feedstock of the CSTR reactors}

128 The inoculum used in this study to start-up the CSTR reactors was obtained from a

129 lab-scale thermophilic $\left(53 \pm 1^{\circ} \mathrm{C}\right) \mathrm{CSTR}$ reactor. Cattle manure, taken from Hashøj

130 biogas plant (Denmark), was used as feedstock. The original manure was sieved with 1

$131 \mathrm{~mm}$ diameter mesh, then stored in a freezer $\left(-21^{\circ} \mathrm{C}\right)$, and thawed in a thermostat $\left(4{ }^{\circ} \mathrm{C}\right)$ 
132 before use. The basic characteristics of the inoculum and the feedstock are shown in

133 Table 1.

\section{$134 \quad 2.2$ Bioaugmentation cultures}

135 Two different bioaugmentation cultures were used in this study. The first culture

136 was an enriched ammonia-tolerant consortium obtained from a previous study (Tian et

137 al., 2019b). It was shown by the $\left[2-{ }^{14} \mathrm{C}\right]$ acetate radioisotopic analyses that aceticlastic

138 methanogenic pathway was dominant in the consortium, which was mediated primarily

139 by Methanosarcina thermophila ( $>95 \%$ abundance among the methanogens). The

140 cultivation of the enriched culture, prior to the bioaugmentation experiments, was

141 conducted in batch bottles (238 and $40 \mathrm{~mL}$ total and working volume, respectively) with

142 basal anaerobic medium (BA medium) and acetate $\left(3.0 \mathrm{~g} \mathrm{HAc}^{-1}\right)$ as the cultivation

143 medium and the main carbon source, respectively. The volatile suspended solid (VSS)

144 concentration was $65 \mathrm{mg} \mathrm{L}^{-1}$ at the end of cultivation. The detailed growth time course

145 of the enriched culture is shown in the E-supplementary data. In order to achieve the

146 same biomass concentrations (final VSS concentration of $332 \mathrm{mg} \mathrm{L}^{-1}$ ) in all

147 bioaugmentation tests, the enriched consortium was up-concentrated five times by

148 centrifuging at $4500 \mathrm{rpm}$ for 10 min under $\mathrm{N}_{2}$ gas headspace.

149 The second bioaugmentation culture was a mixed culture consisting of a pure

150 hydrogenotrophic strain (Methanoculleus thermophilus CR-1 (DSM No. 2373)) and the

151 aforementioned enriched consortium (50/50, based on VSS). It must be mentioned that

152 M. thermophilus and the enriched consortium were cultivated separately, and they were

153 mixed together before the bioaugmentation process. The $M$. thermophilus CR-1 was

154 purchased from DSMZ GmbH Company and cultivated as suggested by DSMZ (2017)

155 with gas mixture of $\mathrm{H}_{2}$ and $\mathrm{CO}_{2}(80 / 20, \mathrm{v} / \mathrm{v})$ as substrate. It was acclimatized to TAN 
156 levels of $5.0 \mathrm{~g} \mathrm{NH}_{4}{ }^{+}-\mathrm{N} \mathrm{L}^{-1}$ at $\mathrm{pH} 8.0$ in batch bottles with 238 and $40 \mathrm{~mL}$ total and

157 working volume, respectively. Moreover, the VSS concentration was determined to be

$158332 \mathrm{mg} \mathrm{L}^{-1}$ at the end of cultivation. The detailed growth of M. thermophilus is shown in

159 the E-supplementary data.

$160 \quad 2.3$ Continuous reactor setup and bioaugmentation strategy

161 Three identical lab-scale CSTR reactors $\left(\mathrm{R}_{\mathrm{ctrl}}, \mathrm{R}_{\mathrm{enr}}\right.$ and $\left.\mathrm{R}_{\text {mix }}\right)$ were used in the

162 current study and each reactor had 2.3 and $1.8 \mathrm{~L}$ total and working volume, respectively.

163 Each CSTR reactor consists of the reactor vessel with a magnetic stirrer, an influent

164 bottle with a stirrer to ensure the substrate homogeneity, a peristaltic pump for feeding,

165 an effluent bottle, an electrical heating jacket and a water-displacement gas meter. The

166 reactors were operating under thermophilic $\left(53 \pm 1^{\circ} \mathrm{C}\right)$ conditions with HRT of 15 days,

167 and organic loading rate (OLR) of $2.50 \mathrm{~g} \mathrm{VS} \mathrm{L}^{-1} \mathrm{~d}^{-1}$ throughout the experiment.

168 The experimental period was divided into four different phases: P1 (days 0-13); P2

169 (days 14-20); P3 (days 21-23) and P4 (days 24-53). The reactors were running at a

170 steady state (less than $10 \%$ methane production variation for at least ten consecutive

171 days (Hansen et al., 1998)) at $\mathrm{P} 1$ with low ammonia levels $\left(1.42 \mathrm{~g} \mathrm{NH}_{4}{ }^{+}-\mathrm{N} \mathrm{L}^{-1}\right)$ in the

172 feedstock. At the beginning of P2, the TAN levels in the reactor were increased to $5.0 \mathrm{~g}$

$173 \mathrm{NH}_{4}{ }^{+}-\mathrm{N} \mathrm{L}^{-1}$ by spiking extra ammonia (ammonium chloride, $\mathrm{NH}_{4} \mathrm{Cl}$, CAS: 12125-02-9,

174 Sigma-Aldrich) into both the reactor and the feedstock, and were kept constant (5.0 $\mathrm{g}$

$\left.175 \mathrm{NH}_{4}{ }^{+}-\mathrm{N} \mathrm{L}^{-1}\right)$ for all following phases (P2-P4). Bioaugmentation was performed in three

176 consecutive days during $\mathrm{P} 3$, with $72 \mathrm{~mL}$ bioaugmentation culture each day in $\mathrm{R}_{\mathrm{enr}}$ (only

177 the enriched culture) and $\mathrm{R}_{\text {mix }}$ (the mixed culture). In total, each reactor was

178 bioaugmented with $216 \mathrm{~mL}$ bioaugmentation inoculum, corresponding to $72 \mathrm{mg}$ dry 
179 biomass. By contrast, $\mathrm{R}_{\text {ctrl }}$ was used as control reactor (abiotic augmentation), where

180 sterile BA medium and pure strain cultivation medium $(50 / 50 \mathrm{v} / \mathrm{v})$ were introduced.

181

182

183

184

185

186

187

188

189

190

191

193

194

195

196

197

198

199

200

201

\subsection{Microbial analyses}

To elucidate how the bioaugmentation culture affected the microbial composition both immediately and long term after bioaugmentation, four triplicate samples were taken from each reactor. The sampling times were at day 6 (before TAN increase, P1), day 17 (after TAN increase but before bioaugmentation, P2), day 26 (immediately after bioaugmentation, $\mathrm{P} 4_{\mathrm{a}}$ ) and day 50 (two HRTs after bioaugmentation, $\mathrm{P} 4_{\mathrm{b}}$ ). Genomic DNA was extracted with DNeasy PowerSoil ${ }^{\circledR}$ Kit (QIAGEN GmbH, Hilden, Germany). Moreover, the DNA concentration and quality were tested by NanoDrop 1000 (Thermofisher). Polymerase chain reaction (PCR) amplification was performed on the $\mathrm{V} 4$ region of $16 \mathrm{~S}$ rRNA gene with universal primers 515F/806R, and high throughput sequencing was performed by Illumina MiSeq platform (Ramaciotti Centre for Genomics, Kensington, Australia).

The raw sequencing reads, which were submitted to Sequence Read Archive (SRA) database (http://www.ncbi.nlm.nih.gov/sra) under the project No.SPR103296, were analysed by CLC Workbench (V.8.0.2) with microbial genomics module plug in (QIAGEN), as was described previously (Tian et al., 2018b). Sequencing depth was ensured by rarefaction curve (E-supplementary data). Furthermore, the taxonomical assignment of the interesting OTUs (relative abundance higher than $0.5 \%$ for bacteria and $0.01 \%$ for archaea, E-supplementary data) was validated through $16 \mathrm{~S}$ ribosomal RNA sequences (Bacteria and Archaea) database on the website of National Center for Biotechnology Information. Furthermore, Multi Experiment Viewer software (MeV 
4.9.0) (Saeed et al., 2006) was used to plot the relative abundance of the interesting

203 OTUs as a heat map.

204

205

206

207

208

209

210

211

212

213

214

215

216

217

218

219

220

221

\subsection{Physicochemical analyses}

TS, VS, VSS, TKN and TAN were determined according to APHA (2012). The $\mathrm{pH}$ was measured by PHM99 LAB pH meter. Methane concentration and VFA levels in the reactors were measured through two different gas-chromatographs as were described before (Tian et al., 2018c).

\subsection{Calculations and statistics}

The OriginLab program (OriginLab Corporation, Northampton, Massachusetts) was used for statistical analyses and the preparation of the figures. The significantly changed $(p<0.05)$ OTUs between different samples were determined by STAMP software (Parks and Beiko, 2010).

\section{Results and discussion}

\subsection{CSTR reactors' performance}

\subsubsection{Methane production efficiency}

During P1, all the reactors performed similarly $(p>0.05)$ achieving a steady state with average methane yields of $163.6 \pm 4.7,165.6 \pm 3.8$ and $167.5 \pm 4.4 \mathrm{~mL} \mathrm{CH}_{4} \mathrm{~g}^{-1} \mathrm{VS}$ for $\mathrm{R}_{\mathrm{ctrl}}, \mathrm{R}_{\mathrm{enr}}$ and $\mathrm{R}_{\mathrm{mix}}$, respectively (Fig. 1a). After TAN levels increased to $5.0 \mathrm{~g} \mathrm{NH}_{4}{ }^{+}-\mathrm{N}$ $\mathrm{L}^{-1}(\mathrm{P} 2)$, the methane yield decreased rapidly and fluctuated with $34-39 \%$ methane production loss compared to the uninhibited $\mathrm{P} 1$. These methane production losses agreed with previous studies reporting that $30-39 \%$ methane reduction was observed in 
223 thermophilic CSTR reactors when TAN levels increased up to $4.9 \mathrm{~g} \mathrm{NH}_{4}^{+}-\mathrm{N} \mathrm{L}^{-1}$ (Sung 224 and Liu, 2003; Tian et al., 2018c).

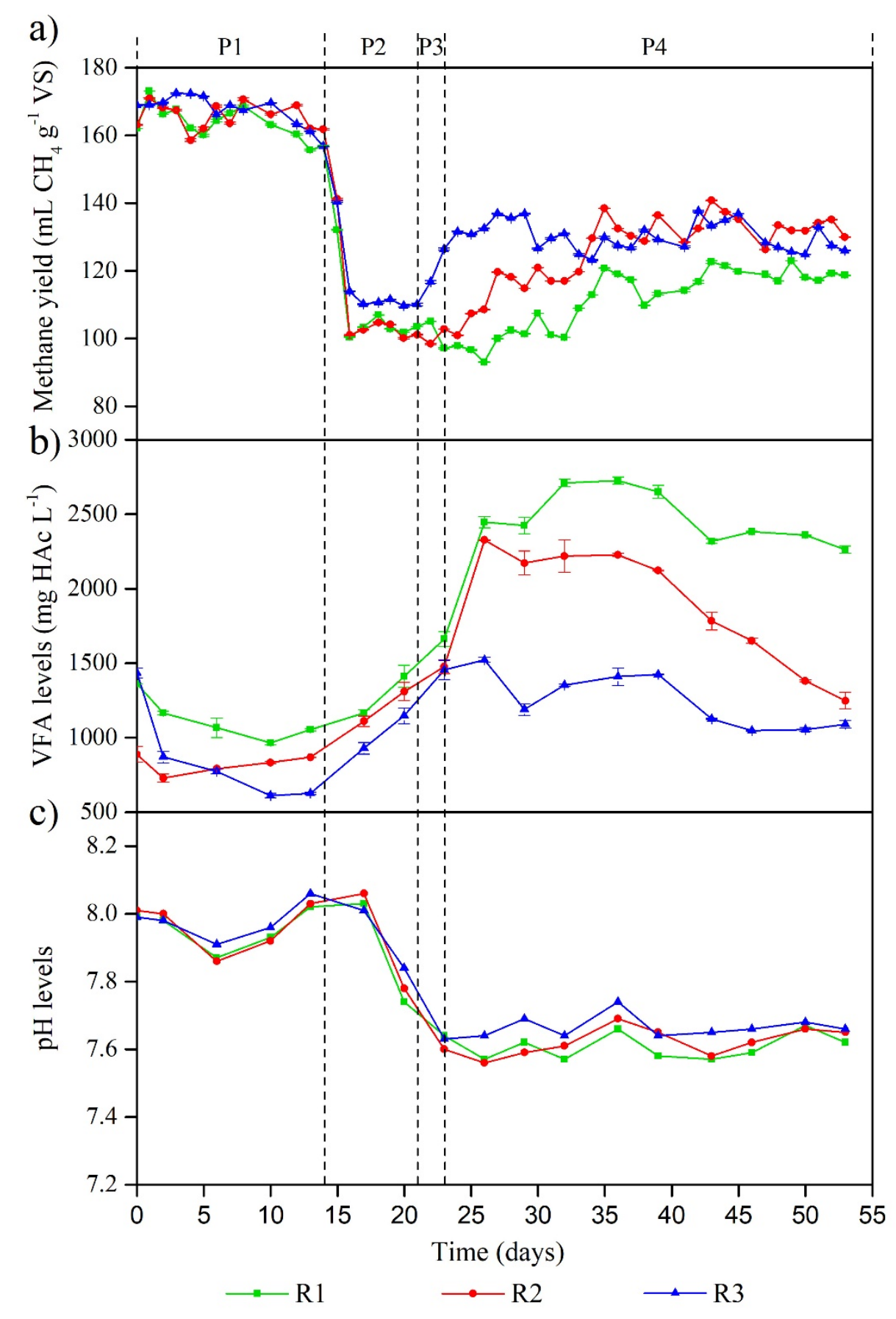

226 Fig. 1. The performance of the three reactors at different experimental phases: a) methane yield, b) total VFA levels and c) $\mathrm{pH}$. P1, P2, P3, P4 stands for the four different experimental phases of the experiment, respectively. 
Immediately after bioaugmentation (P4), the methane production of $\mathrm{R}_{\operatorname{mix}}$

230 (bioaugmentation with the mixed culture) increased by $17 \%$ compared to $\mathrm{P} 2$ and

231 established a steady state with an average yield of $130.3 \pm 4.2 \mathrm{~mL} \mathrm{CH}_{4} \mathrm{~g}^{-1} \mathrm{VS}(78 \%$

232 recovery compared to P1) until the end of the experiment (Fig. 1a). $\mathrm{R}_{\text {enr, }}$ which was

233 bioaugmented with only the enriched consortium, also increased its methane production

234 after bioaugmentation, but it recovered the methane much slower than $\mathrm{R}_{\text {mix }}$.

235 Specifically, the methane production of $\mathrm{R}_{\mathrm{enr}}$ started increasing three days after

236 bioaugmentation, and it became similar to $\mathrm{R}_{\text {mix }}$ after another seven days, with an

237 average methane yield of $133.0 \pm 3.7 \mathrm{~mL} \mathrm{CH}_{4} \mathrm{~g}^{-1} \mathrm{VS}(80 \%$ recovery compared to P1).

238 These results were in accordance with previous studies reporting a sharp methane

239 production increase after bioaugmentation compared to the inhibited period (Fotidis et

240 al., 2014; Fotidis et al., 2017). $\mathrm{R}_{\text {ctrl }}$ also increased its methane yield from $101.3 \pm 3.6 \mathrm{~mL}$

$241 \mathrm{CH}_{4} \mathrm{~g}^{-1}$ VS during P2 to $117.3 \pm 3.8 \mathrm{~mL} \mathrm{CH}_{4} \mathrm{~g}^{-1} \mathrm{VS}$ at the last HRT of the experiment,

242 indicating a certain degree of adaptation of the AD microbiome to the ammonia toxicity,

243 but it was still significantly lower $(p<0.05)$ compared to $\mathrm{R}_{\text {mix }}$ and $\mathrm{R}_{\mathrm{enr}}$. This natural,

244 slow adaptation of methanogenic cultures to high ammonia levels in continuous reactors

245 have also been reported by many researchers (Calli et al., 2005a; Tian et al., 2018b).

246 In the current experiment, $\mathrm{R}_{\mathrm{enr}}$ and $\mathrm{R}_{\text {mix }}$ improved the methane production efficiency

247 by $11-13 \%$ compared to $\mathrm{R}_{\mathrm{ctrl}}$ during the steady state period after bioaugmentation. Thus,

248 for the first time bioaugmentation was applied successfully to alleviate ammonia

249 inhibition in thermophilic CSTR reactors. The similar final improvement of methane

250 production for both bioaugmented reactors $\left(\mathrm{R}_{\operatorname{mix}}\right.$ and $\left.\mathrm{R}_{\mathrm{enr}}\right)$ indicated the comparable

251 levels of the available free energy in the two reactors that the bioaugmented microbiome

252 could utilize at the extreme conditions. It has been reported by Rajagopal et al. (2013) 
253 that high ammonia/ammonium levels could inhibit the methane producing enzyme

254 thereafter increase the energy barrier of the methanogenic reactions. Thus, it seems that

255 the available free energy levels were dictated by the effect of ammonia on the metabolic

256 efficiency of the pre-existing microbiome members, and once the energy barrier was

257 overcome by the bioaugmentation inocula, similar energy was released and recovered.

258 Nonetheless, further investigation using pure carbon sources to pinpoint the free energy

259 availability and the different metabolic bottlenecks are necessary. Another interesting

260 observation was that the methane production was recovered much faster in $\mathrm{R}_{\text {mix }}$ than

$261 \mathrm{R}_{\mathrm{enr}}$, manifested by $15 \%$ higher overall methane production during the first ten days

262 after bioaugmentation. This indicates that the mixed culture was a better

263 bioaugmentation inoculum compared to the enriched culture, which demonstrated the

264 importance of hydrogenotrophic methanogens included in the mixed culture. A possible

265 explanation for this behaviour is that the instant hydrogen partial pressure removal by

266 hydrogenotrophic $M$. thermophilus created better thermodynamic conditions for the

267 overall AD process (Hao et al., 2017).

\subsubsection{VFA accumulation and $\mathrm{pH}$ fluctuation}

In general, the VFA accumulation of each reactor matched with the methane

270 production variations. Specifically, before increasing the ammonia levels, the VFA

271 levels of all the reactors were inside the normal threshold $\left(<1500 \mathrm{mg} \mathrm{L}^{-1}\right)$ (Angelidaki et

272 al., 2005) for CSTR reactors (Fig. 1b). As expected, the VFA levels increased rapidly

273 after increasing ammonia levels and exceeded the $1500 \mathrm{mg} \mathrm{L}^{-1}$ threshold (Angelidaki et

274 al., 2005) at the end of P3 for all the reactors. This VFA increase was reported before

275 when CSTR reactors were exposed to increased ammonia levels (Westerholm et al., 
2012; Tian et al., 2017). Moreover, The VFA increase also supports that

277

methanogenesis was more susceptible to ammonia inhibition compared to acetogenesis.

The VFA levels in the $\mathrm{R}_{\mathrm{ctrl}}$ kept increasing at the beginning of $\mathrm{P} 4$ and then stabilized between 2200 to $2700 \mathrm{mg} \mathrm{L}^{-1}$ throughout the rest of the experiment. In contrast, the VFA increasing trend in $\mathrm{R}_{\text {mix }}$ ceased immediately after bioaugmentation and stabilised around $1000 \mathrm{mg} \mathrm{L}^{-1}$ until the end of the experiment, demonstrating the immediate positive bioaugmentation effect of the mixed culture. While the VFA levels in $\mathrm{R}_{\mathrm{enr}}$ started decreasing three days after bioaugmentation from $2300 \mathrm{mg} \mathrm{L}^{-1}$ to 1250 $\mathrm{mg} \mathrm{L}^{-1}$ at the end of $\mathrm{P} 4$, indicating a slower bioaugmentation effect on VFA reduction. This faster VFA consumption that was observed in $\mathrm{R}_{\text {mix }}$ compared to $\mathrm{R}_{\mathrm{enr}}$, agreed with a previous observation where acetate was consumed faster through SAO-HM (e.g. $\mathrm{R}_{\text {mix }}$ in this study) compared to AM pathway (e.g. $R_{\text {enr }}$ in this study) under stressful conditions (Hao et al., 2011). Moreover, the changes in the accumulations of acetate and propionate (Fig. 2) also supported the faster bioaugmentation effect scenario in $R_{\text {mix }}$ than in $\mathrm{R}_{\mathrm{enr}}$. Specifically, immediately after bioaugmentation, the acetate levels in $\mathrm{R}_{\text {mix }}$ start decreasing followed by the propionate levels. In $\mathrm{R}_{\mathrm{enr}}$, the acetate levels started dropping three days after bioaugmentation and reached similar levels compared to $\mathrm{R}_{\mathrm{mix}}$, while the propionate remained comparatively high but statistically $(p<0.05)$ lower than $\mathrm{R}_{\mathrm{ctrl}}$. Even though the dissolved hydrogen concentration was not directly measured in the current study, it is generally accepted that increasing hydrogen partial pressure due to the methanogenesis inhibition is the main reason for VFA accumulation (especially propionate) during methanogenesis inhibition (Cord-Ruwisch et al., 1997; Björnsson et al., 2001; Boe et al., 2010). Therefore, the VFA levels decreased in both reactors $\left(\mathrm{R}_{\mathrm{mix}}\right.$ and $\mathrm{R}_{\mathrm{enr}}$ ) after bioaugmentation since aceticlastic $M$. thermophila was present. However, 
300 due to the additional presence of hydrogenotrophic $M$. thermophilus in $\mathrm{R}_{\text {mix }}$, the reactor

301 had a faster hydrogen partial pressure reduction compared to $\mathrm{R}_{\mathrm{enr}}$, which resulted in an

302 even faster VFA reduction. Additionally, this direct hydrogen consumption cut off the

303 back step reaction (homoacetogenesis- accumulation of acetate) and made syntrophic

304 acetate oxidation and other VFAs degradation processes (Eq. 1-5) thermodynamically

305 favourable (Massé and Droste, 2000). To sum up, thermodynamic conditions of the

306 overall AD process were improved through bioaugmentation, consequently increased

307 the methane production and decreased the VFA accumulation. Overall, at the end of the

308 experiment, the bioaugmented reactors, i.e. $\mathrm{R}_{\mathrm{enr}}$ and $\mathrm{R}_{\mathrm{mix}}$, had $45-52 \%$ lower VFA levels

309 compared to $\mathrm{R}_{\mathrm{ctrl}}$ reactor.

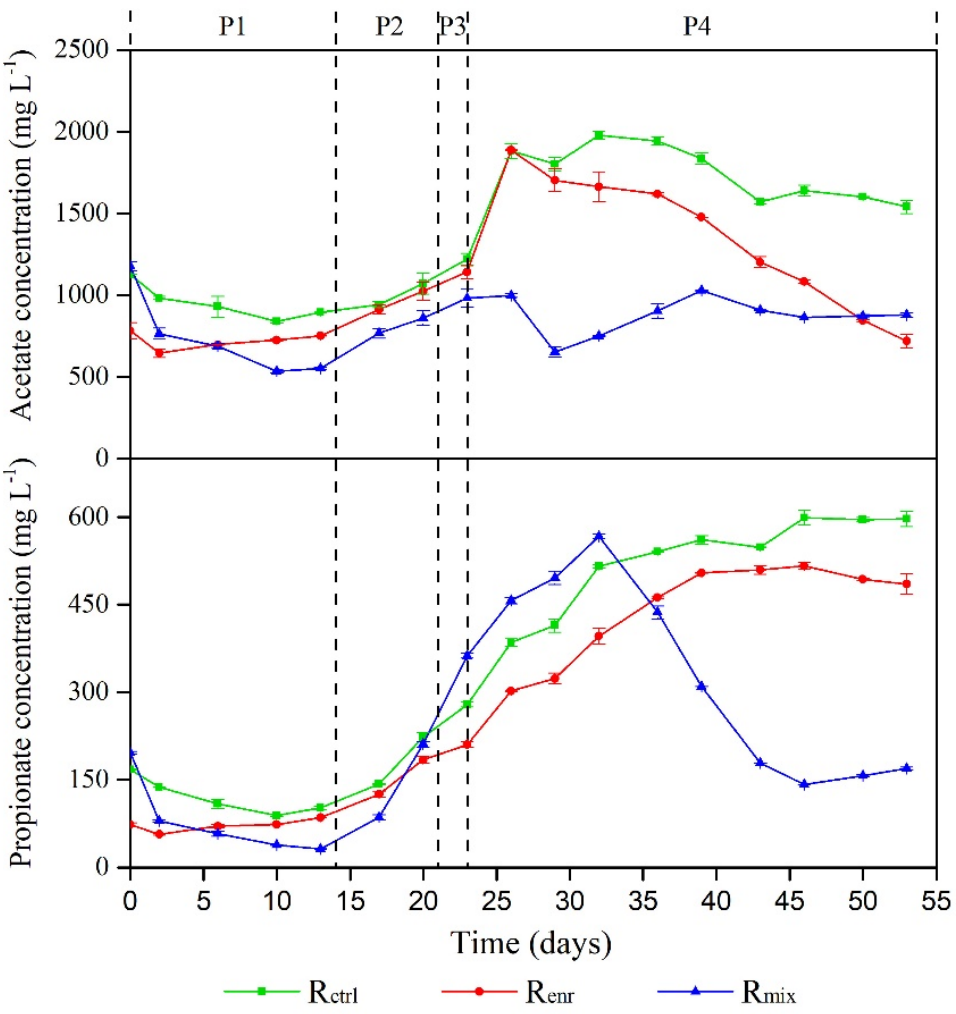

311 Fig. 2. The changes of the individual VFA at different experimental phases: a) acetate

312 levels and b) propionate levels. P1, P2, P3, P4 stands for the four different

313 experimental phases of the experiment, respectively. 
315 (P1, Fig. 1c), and decreased to lower levels varying between 7.55 and 7.75 due to the

316 VFA accumulation after ammonia addition (P2-P4) (Angelidaki and Ahring, 1993).

317 However, thanks to the high buffer capacity of manure, even with fluctuations, the $\mathrm{pH}$

318 levels remained within the optimal $\mathrm{pH}$ range (6.5-8.5) for AD process (Nissilä et al., 319 2012).

\subsection{Microbial community composition and dynamics}

\subsubsection{Microbiome overview}

322 The alpha diversity (based on Chao 1 bias-corrected index (Zhu et al., 2017)) in

$323 \mathrm{R}_{\text {mix }}$ decreased throughout the experiment to form a more specialized community (Fig.

3243 ), which resulted in a more efficient AD process. However, the alpha diversity in $R_{\text {ctrl }}$

325 and $\mathrm{R}_{\mathrm{enr}}$ increased at $\mathrm{P} 2$ and $\mathrm{P} 4 \mathrm{a}$ compared to $\mathrm{P} 1$; it seems that ammonia addition

326 stimulated the growth of microbes that were less abundant during P1 and resulted in a

327 temporarily high diversity (Werner et al., 2014). For both reactors, the diversity

328 decreased at the end of $\mathrm{P} 4$, where species were eliminated from the reactors (washout 329 effect (Tian et al., 2018b)) due to ammonia toxicity stress and more specialised

330 communities were formed.

331

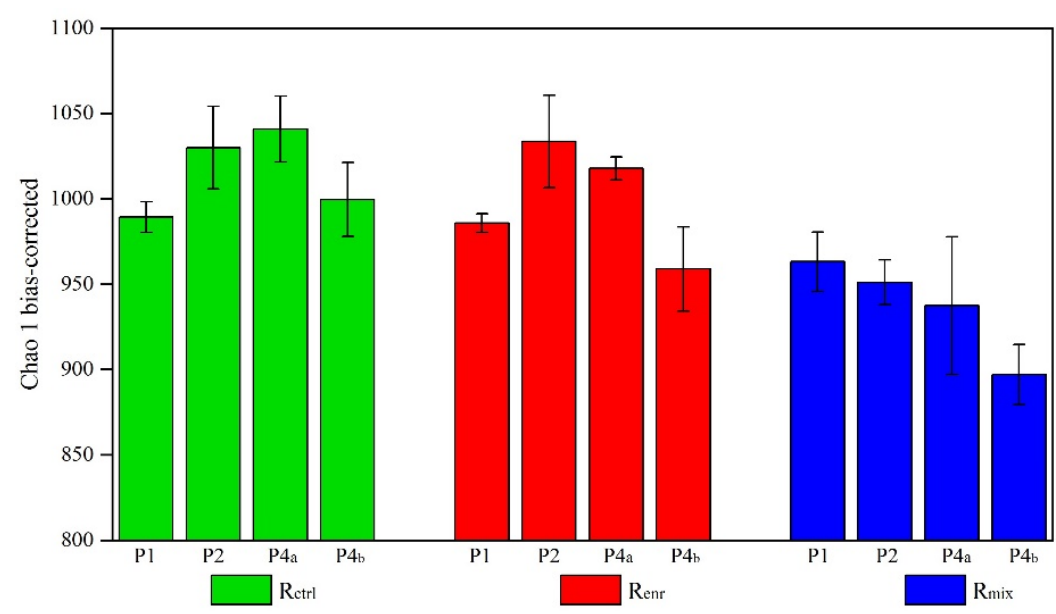


Fig. 3. Alpha diversity based on Chao 1 bias-corrected index at different phases of the experiment.

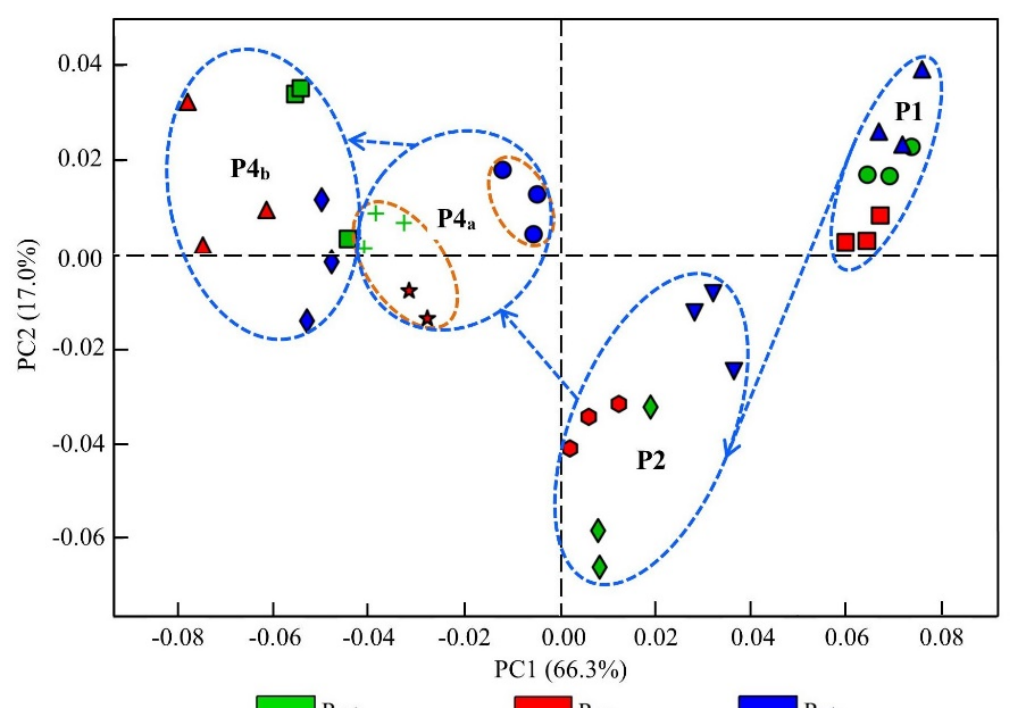

335 Fig. 4. Principal component analysis of the triplicate samples from different phases and reactors. PC1 and PC2 stand for principal component 1 and 2, which explain $66.3 \%$ and $17.0 \%$ of the community variation, respectively. $\mathrm{P} 1, \mathrm{P} 2, \mathrm{P} 4_{\mathrm{a}}$ and $\mathrm{P} 4_{\mathrm{b}}$ stands for the four sampling points, respectively: P1 at day 6 (before TAN increase), P2 at day 17 (after TAN increase but before bioaugmentation), $\mathrm{P} 4_{\mathrm{a}}$ at

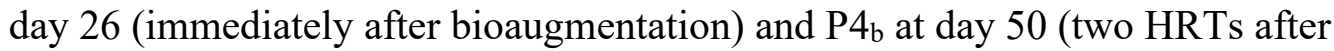
Beta diversity based on principal component analysis (Fig. 4) showed clear

343 microbial community dynamicity between the different experimental phases. At P1, all

344 the samples clustered together indicating a high similarity of the microbial community 345 of all the reactors. However, after the addition of ammonia, the microbial composition 346 in all reactors started differentiating compared to P1. Specifically, an interesting finding 347 was that immediately after bioaugmentation $\left(\mathrm{P} 4_{\mathrm{a}}\right)$, the triplicate samples from $\mathrm{R}_{\mathrm{mix}}$ 
348 clustered together and were significantly different from $\mathrm{R}_{\mathrm{ctrl}}(p<0.05)$ and $\mathrm{R}_{\mathrm{enr}}(p<0.05)$.

349 This difference might contribute to the better performance of $\mathrm{R}_{\text {mix }}$ compared to $\mathrm{R}_{\text {ctrl }}$ and

$350 \mathrm{R}_{\mathrm{enr}}$ during that period. Overall, it was clear also by the current experiment that

351 ammonia plays a decisive role in shaping anaerobic microbial communities (De Vrieze

352 et al., 2015; Tian et al., 2018b); and that a new, ammonia-tolerant microbiome could be

353 established rapidly through bioaugmentation (Fotidis et al., 2017) based on the

354 improved reactor performance.

355

356

357

358

359

360

361

362

363

364

365

366

367

\subsubsection{Communities dynamics}

In general, the microbial community consisted of mainly bacteria, which accounted for more than $97 \%$ of the total relative abundance, and the rest were archaea. Firmicutes was the predominant phylum in all the samples (the relative abundance varied from $73 \%$ to $78 \%$ ), followed by Bacteroidetes (13-15\%), and other less abundant phyla, such as Proteobacteria, Synergistetes, Thermotogae, Euryarchaeota, etc. (E-supplementary data).

Regarding the changes of each individual OTU (Fig. 5), the first noteworthy OTU was M. thermophilus 2, which represented the hydrogenotrophic Methanoculleus thermophilus that was included in the mixed bioaugmentation culture. Its relative abundance decreased significantly $(p<0.05)$ in all reactors after ammonia addition (Fig. 5a-c). The decreased low abundance remained similarly during the rest periods in $\mathrm{R}_{\mathrm{ctrl}}$ until the end of the experiment and increased slightly in $\mathrm{R}_{\mathrm{enr}}$ to $2.9 \%$ (with respect to the archaea community) at $\mathrm{P}_{4 b}$, while it increased more than twofold in $\mathrm{R}_{\text {mix }}$ and reached $11.5 \%$ at $\mathrm{P}_{\mathrm{b}}$ after bioaugmentation. At the same time, an interesting observation was that two OTUs, Thermoanaerobacteraceae sp.17 and Thermoanaerobacteraceae sp.18, which were identified to be Thermacetogenium phaeum (93\% similarity), decreased 
372 significantly $(p<0.05)$ in all the three reactors after the addition of ammonia. However,

373 it increased significantly in $\mathrm{R}_{\text {mix }}$ by $32 \%$ immediately after bioaugmentation, and

374 reached a significant higher relative abundance (6.5\%) compared to $\mathrm{R}_{\mathrm{ctrl}}$ and $\mathrm{R}_{\mathrm{enr}} . T$.

375 phaeum is a well-known syntrophic acetate oxidising bacterium (SAOB) (Hattori et al.,

376 2000), which couples with hydrogenotrophic methanogens to mediate the SAO-HM

377 pathway. Therefore, the current dynamics of the three aforementioned OTUs indicated

378 that bioaugmentation with hydrogenotrophic $M$. thermophilus in $\mathrm{R}_{\text {mix }}$ stimulated the

379 growth of $T$. phaeum thus resulted in faster syntrophic acetate oxidation (SAO) process

380 and consequently recovered the methane production faster than the other two reactors.

381 The result also highlighted the importance of hydrogenotrophic methanogens in the

382 bioaugmentation process.

383 On the other hand, the relative abundance of Methanosarcina thermophila

384 (dominant methanogen in the bioaugmented enriched culture) was very low in all

385 reactors before bioaugmentation. After bioaugmentation, it increased significantly

$386(p<0.05)$ by more than twofold in $\mathrm{R}_{\mathrm{enr}}$ and $\mathrm{R}_{\mathrm{mix}}$, but not in $\mathrm{R}_{\mathrm{ctrl}}$, verifying the successful

387 establishment of the selected cultures in the continuous reactors through

388 bioaugmentation. However, the high abundance of $M$. thermophila was detected only at

389 P4 $4_{a}$ (i.e. short period immediately after bioaugmentation), and it almost disappeared at

390 the end of the experiment. Methanosarcina spp. play an important role in consuming

391 acetate when acetate accumulates in thermophilic reactors (Hori et al., 2006). Therefore,

392 in the current study, it seems that M. thermophila thrived during the acetate

393 accumulation period, and it was washed out due to interspecific competition (Tian et al.,

394 2018b) when the acetate levels went below $1000 \mathrm{mg} \mathrm{HAc} \mathrm{L}^{-1}$ (E-supplementary data). 
395 As a result, the enriched consortium triggered the establishment of the new and 396 specialised community with efficient biogas productivity at high ammonia levels.

397 Throughout the experimental period, the most dominant methanogenic OTU was 398 always Methanothermobacter sp.1 in all three reactors. The relative abundance of 399 Methanothermobacter sp.1 varied between 60 to $83 \%$ (total methanogens) at different 400 phases (Fig. 5a-c), verifying a well-documented strong hydrogenotrophic methanogenic 401 activity of Methanothermobacter spp. in thermophilic reactors (Yabu et al., 2011; 402 Franke-Whittle et al., 2014; Kougias et al., 2017). Moreover, the relative abundance of 403 Methanothermobacter sp.1 decreased significantly $(p<0.05)$ after the addition of 404 ammonia, but it increased back during P4 up to the initial levels (P1) for reactors $\mathrm{R}_{\mathrm{ctrl}}$ 405 and $R_{\text {enr, }}$ indicating its ability to adapt at high ammonia levels (Niu et al., 2013).

406 However, although Methanothermobacter sp. kept being dominant, its relative 407 abundance in $\mathrm{R}_{\text {mix }}$ decreased significantly $(p<0.05)$ at $\mathrm{P} 4_{\mathrm{b}}$, which could be explained by 408 the increased abundance of bioaugmented Methanoculleus thermophilus, as discussed 409 above. 
a)
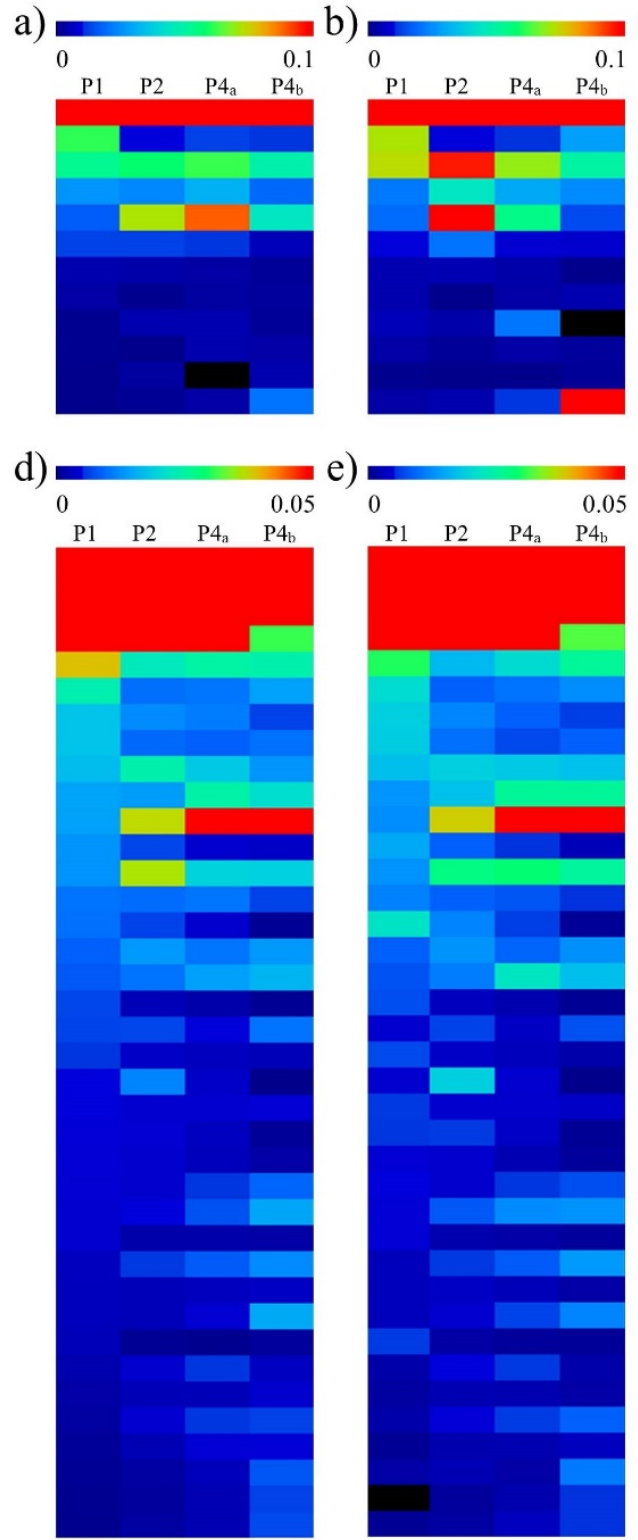

c)

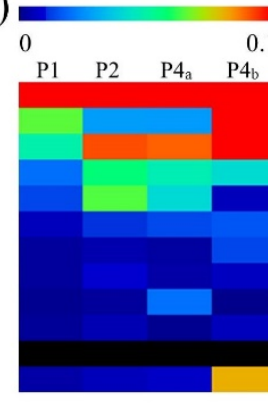

\section{Archaea}

Methanothermobacter sp. 1 Methanoculleus thermophilus 2 Methanobrevibacter millerae 3 Methanobrevibacter olleyae 4 Methanosarcina sp.5

Methanobrevibacter boviskoreani 6

Methanobrevibacter oralis 7

Methanomassiliicoccus sp. 8

Methanosarcina thermophila 9

Methanosphaera stadtmanae 10

Methanospirillum sp.11

Methanobrevibacter sp. 12

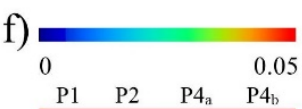

\section{Bacteria}

Clostridiaceae sp.13

Bacteroidia sp.14

Clostridiaceae sp.15

Thermoanaerobacteraceae sp. 17

Thermoanaerobacteraceae sp.18

Thermoanaerobacteraceae sp.19

Anaerobaculum mobile sp. 20

Clostridiales sp. 21

Clostridium sp.22

Halanaerobiaceae sp. 23

Bacillales sp. 24

Thermoanaerobacteraceae sp. 25

Thermoanaerobacteraceae sp. 26

Syntrophomonadaceae sp.27

Defluviitoga tunisiensis 28

Herbivorax saccincola 29

Clostridiales sp. 30

Pseudomonas caeni sp.31

Dethiobacter sp.32

Marinilabiliaceae sp.33

Tepidanaerobacter sp. 34

Synergistaceae sp.35

Synergistaceae sp.36

Thermoanaerobacteraceae sp. 37

Clostridiaceae sp. 38

Caldicoprobacteraceae sp.39

Clostridium caenicola 40

Romboutsia timonensis 41

Ramboutsia timoneae $\mathrm{sp} .42$

Eubacteriaceae sp.43

Bacillaceae sp. 44

Desulfobacterales sp.46

Fermentimonas caenicola 47

Oligella ureolytica 48

Oligella ureolytica 48
Spirochaetaceae sp. 49

MBA08 sp.50
Synergistaceae sp.16

Corynebacterium pollutisoli 45

Fig. 5. Relative abundance shown as heat map for the interesting archaea and bacteria at

415 relative abundance by more than twofold after ammonia addition in all the reactors. This

416 could be due to the ability of Methanosarcina spp. to form aggregates, which might

417 create an ammonia gradient to the core of the aggregate, thus reduce the ammonia 
418 toxicity to some extent (Calli et al., 2005b; Goberna et al., 2010). However, the rapid

419 decreased relative abundance of Methanosarcina sp.5 during the following phases

420 demonstrated its limited long-term tolerance to high ammonia toxicity.

421 Finally, the bioaugmentation also triggered changes of the most abundant bacteria 422 group, i.e. the uncultured MBA08 that included Clostridiaceae sp.13, Clostridiaceae 423 sp.15, Clostridiales sp.21 and MBA08 sp.50. The relative abundance of MBA08 424 increased slightly from $30 \%$ to $31 \%$ in $\mathrm{R}_{\mathrm{ctr}}$, but significantly from $31 \%$ to $34 \%$ in $\mathrm{R}_{\mathrm{enr}}$ 425 and from $28 \%$ to $35 \%$ in $\mathrm{R}_{\text {mix }}$, before and after bioaugmentation, respectively. MBA08 426 was previously reported to be dominant in thermophilic reactors operated at high 427 ammonia levels (De Vrieze et al., 2015) and was suspected to involve in cellulose 428 degradation (Sun et al., 2015). It seems that the high fibre content in the feedstock 429 (cattle manure) contribute to the high abundance of MBA08 in this study, and the 430 significant increase of its abundance in $\mathrm{R}_{\mathrm{enr}}$ and $\mathrm{R}_{\operatorname{mix}}$ was in accordance with the high 431 methane production after bioaugmentation. However, the aforementioned discussion 432 was derived by a DNA-based methodology (i.e. 16r rRNA gene sequencing) and as De

433 Vrieze et al. (2016) has pointed out, the microbial abundance does not necessarily 434 reflects the microbial activity. Thus more advanced microbiology, such as RNA-based 435 metatranscriptomics, is suggested for future studies correlating microbial community 436 and activity.

\section{$437 \quad 3.3 \quad$ A proposed mechanism of the bioaugmentation effect}

438 Overall, the immediate reduction of the hydrogen partial pressure by the

439 bioaugmented hydrogenotrophic methanogen seemed to be the main reason for a fast

440 and more efficient bioaugmentation. Even though both bioaugmentation inocula

441 resulted in an improved reactor performance, the mixed culture had a faster effect in 
442 recovering the methane production compared to the enriched culture, which indicated

443 that the hydrogenotrophic $M$. thermophilus was the key to a successful bioaugmentation

444 effect.
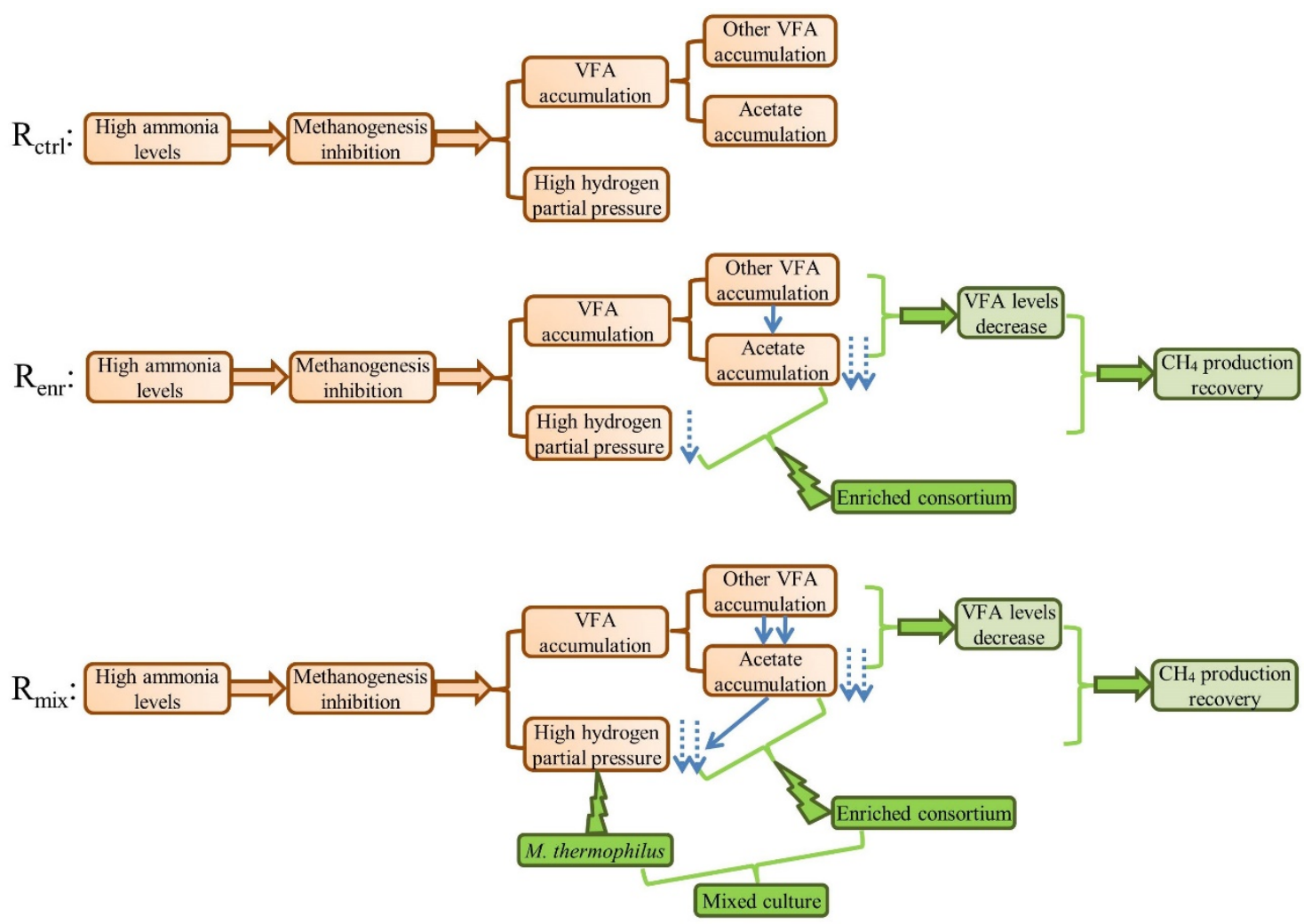

446 Fig. 6. Proposed mechanisms depicting the effects of bioaugmentation on the three reactors. The blue solid and dashed arrows present the conversion direction and decrease of the compounds' concentration, respectively, and two arrows stand for stronger effect compared to the single arrow. The orange and green processes stand for the reactors' performance before and after bioaugmentation, respectively.

Based on the evaluation of all operational and microbiological data, a potential 453 bioaugmentation working mechanism was proposed (Fig. 6). Specifically, the instantly decreased hydrogen partial pressure, due to the consumption by $M$. thermophilus in 
$455 \mathrm{R}_{\text {mix }}$, ceased homoacetogenesis, increased SAO and created favourable conditions (Eq.

456 (1), (2), (4)) for VFA degradation (Massé and Droste, 2000; Hao et al., 2017) by

457 enhancing acetogenesis (especially from propionate, E-supplementary data).

458 Meanwhile, the enriched consortium (mainly aceticlastic $M$. thermophila) in the mixed

459 culture also degraded acetate directly. Therefore, the VFA-ammonia synergistic

460 inhibitory effect (Lu et al., 2013) was alleviated rapidly due to the reduction of the

461 hydrogen partial pressure, which resulted in a faster methane recovery compared to the

462 other reactors. When only the enriched culture was used ( $\left.\mathrm{R}_{\mathrm{enr}}\right)$, it seems that it mainly

463 decreased acetate degradation (Fig. 2a) through AM pathway, but it did not significantly

464 contributed to the hydrogen partial pressure reduction. This resulted in a slower

465 acetogenic step from other VFAs. For example, propionate levels did not decrease

466 significantly in $\mathrm{R}_{\mathrm{enr}}$ after bioaugmentation (Fig. 2b). Therefore, both strategies

467 alleviated ammonia inhibition, but the bioaugmentation only with the enriched culture

468 did not recover the methane production as fast as the bioaugmentation with the mixed

469 culture.

\section{Conclusions}

471 The results demonstrated the basic mechanism that drives a successful and fast

472 bioaugmentation process in ammonia inhibited CSTR reactors. This mechanism

473 includes/requires the instant hydrogen partial pressure reduction by bioaugmented

474 ammonia-tolerant hydrogenotrophic methanogens that allows a thermodynamic SAO

475 process, which consequently decreases the VFA levels (reduction of the VFA-ammonia

476 synergistic inhibitory effect) and thus, improves the overall AD process. Furthermore, it

477 showed for the first time that bioaugmentation with ammonia-tolerant cultures can

478 alleviate ammonia inhibition in thermophilic continuous reactors. Overall, the 
479 comparison between the different bioaugmentation inocula highlighted the crucial role

480 of hydrogenotrophic methanogen in this process.

\section{Acknowledgements}

482 This work was supported by Energinet.dk under the project framework ForskEL

483 "MicrobStopNH $\mathrm{N}_{3}$-Innovative bioaugmentation strategies to tackle ammonia inhibition in 484 anaerobic digestion process" (program no. 2015-12327). Hailin Tian would like to 485 thank China Scholarship Council for the financial support. The authors thank Hector 486 Garcia for his technical support and Hao Sun for providing inoculum for this study.

\section{Appendix A. Supplementary material}

E-supplementary data of this work can be found in online version of the paper.

\section{References}

490 [1] Angelidaki, I., Ahring, B., 1993. Thermophilic anaerobic digestion of livestock waste: the effect of ammonia. Appl. Microbiol. Biotechnol. 38, 560-564.

492 [2] Angelidaki, I., Boe, K., Ellegaard, L., 2005. Effect of operating conditions and reactor configuration on efficiency of full-scale biogas plants. Water Sci. Technol. 52, 189-194.

495

[3] Angelidaki, I., Karakashev, D., Batstone, D.J., Plugge, C.M., Stams, A.J., 2011. Biomethanation and its potential. Methods Enzymol. 494, 327-51.

497 [4] APHA, 2012. Standard Methods for the Examination of Water and Wastewater. American Public Health Association, Washington, DC. 
[5] Björnsson, L., Murto, M., Jantsch, T.G., Mattiasson, B., 2001. Evaluation of new methods for the monitoring of alkalinity, dissolved hydrogen and the microbial community in anaerobic digestion. Water Res. 35, 2833-2840.

[6] Boe, K., Batstone, D.J., Steyer, J.-P., Angelidaki, I., 2010. State indicators for monitoring the anaerobic digestion process. Water Res. 44, 5973-5980.

[7] Calli, B., Mertoglu, B., Inanc, B., Yenigun, O., 2005a. Effects of high free ammonia concentrations on the performances of anaerobic bioreactors. Process Biochem. 40, 1285-1292.

[8] Calli, B., Mertoglu, B., Inanc, B., Yenigun, O., 2005b. Methanogenic diversity in anaerobic bioreactors under extremely high ammonia levels. Enzyme Microb. Technol. 37, 448-455.

[9] Cord-Ruwisch, R., Mercz, T.I., Hoh, C.-Y., Strong, G.E., 1997. Dissolved hydrogen concentration as an on-line control parameter for the automated operation and optimization of anaerobic digesters. Biotechnol. Bioeng. 56, 626-634.

[10] De Vrieze, J., Regueiro, L., Props, R., Vilchez-Vargas, R., Jáuregui, R., Pieper, D.H., Lema, J.M., Carballa, M., 2016. Presence does not imply activity: DNA and RNA patterns differ in response to salt perturbation in anaerobic digestion. Biotechnol. Biofuels 9, 244.

[11] De Vrieze, J., Saunders, A.M., He, Y., Fang, J., Nielsen, P.H., Verstraete, W., Boon, N., 2015. Ammonia and temperature determine potential clustering in the anaerobic digestion microbiome. Water Res. 75, 312-323.

[12] DSMZ. 2017. 141. Methanogenium medium $\left(\mathrm{H}_{2} / \mathrm{CO}_{2}\right)$, Leibniz-Institut DSMZ - Deutsche Sammlung von Mikroorganismen und Zellkulturen GmbH. 
523 [13] Fotidis, I.A., Treu, L., Angelidaki, I., 2017. Enriched ammonia-tolerant methanogenic cultures as bioaugmentation inocula in continuous biomethanation processes. J. Cleaner Prod. 166, 1305-1313.

526 [14] Fotidis, I.A., Wang, H., Fiedel, N.R., Luo, G., Karakashev, D.B., Angelidaki, I., 2014. Bioaugmentation as a solution to increase methane production from an ammonia-rich substrate. Environ. Sci. Technol. 48, 7669-76.

[15] Franke-Whittle, I.H., Walter, A., Ebner, C., Insam, H., 2014. Investigation into the effect of high concentrations of volatile fatty acids in anaerobic digestion on methanogenic communities. Waste Manage. 34, 2080-2089.

[16] Goberna, M., Gadermaier, M., García, C., Wett, B., Insam, H., 2010. Adaptation of methanogenic communities to the cofermentation of cattle excreta and olive mill wastes at $37 \mathrm{C}$ and 55 C. Appl. Environ. Microbiol. 76, $6564-6571$.

[17] Hansen, K.H., Angelidaki, I., Ahring, B.K., 1998. Anaerobic digestion of swine manure: inhibition by ammonia. Water Res. 32, 5-12.

538 [18] Hao, L.-P., Lü, F., He, P.-J., Li, L., Shao, L.-M., 2011. Predominant Contribution of Syntrophic Acetate Oxidation to Thermophilic Methane Formation at High Acetate Concentrations. Environ. Sci. Technol. 45, 508513.

542 [19] Hao, X., Liu, R., van Loosdrecht, M.C.M., Cao, D., 2017. Batch influences of exogenous hydrogen on both acidogenesis and methanogenesis of excess sludge. Chem. Eng. J. 317, 544-550. 
545 [20] Hattori, S., Kamagata, Y., Hanada, S., Shoun, H., 2000. Thermacetogenium

546 phaeum gen. nov., sp. nov., a strictly anaerobic, thermophilic, syntrophic

547 acetate-oxidizing bacterium. Int. J. Syst. Evol. Microbiol. 50 Pt 4, 1601-1609.

548 [21] Hori, T., Haruta, S., Ueno, Y., Ishii, M., Igarashi, Y., 2006. Dynamic

549 Transition of a Methanogenic Population in Response to the Concentration of

$550 \quad$ Volatile Fatty Acids in a Thermophilic Anaerobic Digester. Appl. Environ.

$551 \quad$ Microbiol. 72, 1623-1630.

552 [22] Kamali, M., Gameiro, T., Costa, M.E.V., Capela, I., 2016. Anaerobic digestion

553 of pulp and paper mill wastes - An overview of the developments and

554 improvement opportunities. Chem. Eng. J. 298, 162-182.

555 [23] Kim, M., Ahn, Y.-H., Speece, R.E., 2002. Comparative process stability and

556 efficiency of anaerobic digestion; mesophilic vs. thermophilic. Water Res. 36,

$557 \quad 4369-4385$.

558 [24] Kougias, P.G., Treu, L., Benavente, D.P., Boe, K., Campanaro, S., Angelidaki,

559 I., 2017. Ex-situ biogas upgrading and enhancement in different reactor

$560 \quad$ systems. Bioresour. Technol. 225, 429-437.

561 [25] Lu, F., Hao, L., Guan, D., Qi, Y., Shao, L., He, P., 2013. Synergetic stress of

562 acids and ammonium on the shift in the methanogenic pathways during

563 thermophilic anaerobic digestion of organics. Water Res. 47, 2297-306.

564 [26] Massé, D.I., Droste, R.L., 2000. Comprehensive model of anaerobic digestion

565 of swine manure slurry in a sequencing batch reactor. Water Res. 34, 3087-

$566 \quad 3106$. 
[27] Massé, D.I., Rajagopal, R., Singh, G., 2014. Technical and operational feasibility of psychrophilic anaerobic digestion biotechnology for processing ammonia-rich waste. Appl. Energy 120, 49-55.

Möller, K., Müller, T., 2012. Effects of anaerobic digestion on digestate nutrient availability and crop growth: A review. Eng. Life Sci. 12, 242-257.

[29] Nissilä, M.E., Li, Y.-C., Wu, S.-Y., Lin, C.-Y., Puhakka, J.A., 2012. Hydrogenic and methanogenic fermentation of birch and conifer pulps. Appl. Energy 100, 58-65.

[30] Niu, Q., Qiao, W., Qiang, H., Li, Y.-Y., 2013. Microbial community shifts and biogas conversion computation during steady, inhibited and recovered stages of thermophilic methane fermentation on chicken manure with a wide variation

[31] Parks, D.H., Beiko, R.G., 2010. Identifying biologically relevant differences between metagenomic communities. Bioinformatics 26, 715-721.

[32] Rajagopal, R., Massé, D.I., Singh, G., 2013. A critical review on inhibition of anaerobic digestion process by excess ammonia. Bioresour. Technol. 143, 632641.

[33] Saeed, A.I., Bhagabati, N.K., Braisted, J.C., Liang, W., Sharov, V., Howe,

[34] Scarlat, N., Dallemand, J.-F., Fahl, F., 2018. Biogas: Developments and perspectives in Europe. Renew. Energy 129, 457-472. 
[35] Sun, L., Müller, B., Westerholm, M., Schnürer, A., 2014. Syntrophic acetate oxidation in industrial CSTR biogas digesters. J. Biotechnol. 171, 39-44.

[36] Sun, L., Pope, P.B., Eijsink, V.G.H., Schnürer, A., 2015. Characterization of microbial community structure during continuous anaerobic digestion of straw and cow manure. Microb. Biotechnol. 8, 815-827.

[37] Sung, S., Liu, T., 2003. Ammonia inhibition on thermophilic anaerobic digestion. Chemosphere 53, 43-52.

597

[38] Tian, H., Fotidis, I.A., Kissas, K., Angelidaki, I., 2018a. Effect of different ammonia sources on aceticlastic and hydrogenotrophic methanogens. Bioresour. Technol. 250, 390-397.

600 [39] Tian, H., Fotidis, I.A., Mancini, E., Angelidaki, I., 2017. Different cultivation 601 methods to acclimatise ammonia-tolerant methanogenic consortia. Bioresour. Technol. 232, 1-9.

603

[40] Tian, H., Fotidis, I.A., Mancini, E., Treu, L., Mahdy, A., Ballesteros, M., González-Fernández, C., Angelidaki, I., 2018b. Acclimation to extremely high ammonia levels in continuous biomethanation process and the associated microbial community dynamics. Bioresour. Technol. 247, 616-623.

607 [41] Tian, H., Karachalios, P., Angelidaki, I., Fotidis, I.A., 2018c. A proposed mechanism for the ammonia-LCFA synergetic co-inhibition effect on anaerobic digestion process. Chem. Eng. J. 349, 574-580.

610 [42] Tian, H., Mancini, E., Treu, L., Angelidaki, I., Fotidis, I.A., 2019a. Bioaugmentation strategy for overcoming ammonia inhibition during biomethanation of a protein-rich substrate. Chemosphere 231, 415-422. 
613 [43] Tian, H., Treu, L., Konstantopoulos, K., Fotidis, I.A., Angelidaki, I., 2019b. 16s rRNA gene sequencing and radioisotopic analysis reveal the composition of ammonia acclimatized methanogenic consortia. Bioresour. Technol. 272, 54-62.

617 [44] Weiland, P., 2010. Biogas production: current state and perspectives. Appl. Microbiol. Biotechnol. 85, 849-860.

[45] Werner, J.J., Garcia, M.L., Perkins, S.D., Yarasheski, K.E., Smith, S.R., Muegge, B.D., Stadermann, F.J., DeRito, C.M., Floss, C., Madsen, E.L., Gordon, J.I., Angenent, L.T., 2014. Microbial Community Dynamics and Stability during an Ammonia-Induced Shift to Syntrophic Acetate Oxidation. Appl. Environ. Microbiol. 80, 3375.

[46] Westerholm, M., Leven, L., Schnurer, A., 2012. Bioaugmentation of syntrophic acetate-oxidizing culture in biogas reactors exposed to increasing levels of ammonia. Appl. Environ. Microbiol. 78, 7619-25.

[47] Yabu, H., Sakai, C., Fujiwara, T., Nishio, N., Nakashimada, Y., 2011. Thermophilic two-stage dry anaerobic digestion of model garbage with ammonia stripping. J. Biosci. Bioeng. 111, 312-319.

630 [48] Yenigün, O., Demirel, B., 2013. Ammonia inhibition in anaerobic digestion: A review. Process Biochem. 48, 901-911.

632 [49] Zhu, X., Treu, L., Kougias, P.G., Campanaro, S., Angelidaki, I., 2017. Characterization of the planktonic microbiome in upflow anaerobic sludge blanket reactors during adaptation of mesophilic methanogenic granules to thermophilic operational conditions. Anaerobe 46, 69-77. 